\title{
NOTAS SOBRE EL GÉNERO OUDEMANSIELLA SPEG. EN ANDALUCÍA
}

\author{
Antonio ORTEGA, María Teresa VIZOSO y María del Mar ZEA
}

\begin{abstract}
RESUMEN. Se realiza un estudio sobre cuatro especies del género Oudemansiella Speg. procedentes de diversos puntos geográficos de Andalucía. De entre ellos destacan $O$. mediterranea (Pacioni \& Lalli) Horak cuyo encuadramiento taxonómico se discute y $O$. xeruloides Bon siendo destacables nuestras recolectas ya que se separan claramente de la especie tipo por su hábitat no sabulícola.
\end{abstract}

Palabras clave. Taxonomía, Oudemansiella (Agaricales, Basidiomycotina), Andalucía (España).

SUMMARY. A study about four species of the genus Oudemansiella Speg., coming from several geographics places from Andalucia, are done. O. mediterranea (Pacioni \& Lalli) Horak and $O$. xeruloides Bon stand out: the first one by its taxonomic treatment which is discussed and the second one because of our recolections are separated of the specie type for the non sabulicolous habitat.

Key words. Taxonomy, Oudemansiella (Agaricales, Basidiomycotina), Andalucía (España).

\section{INTRODUCCIÓN}

El género Oudemansiella Speg. ha sido objeto en los últimos años de numerosas publicaciones dado el diferente tratamiento taxonómico que, dependiendo de los autores, han recibido algunas de sus especies. De forma breve y a modo de resumen podemos indicar que micólogos como Moser (1983), Pegler \& Young (1986), Horak (1988)... etc. utilizan el sentido clásico del género diferenciando el subgénero Xerula (Mre.) Sing. para aquellas especies con cutícula seca y el estipe cubierto de pelos velutinos de color castaño oscuro, y Oudemansiella Speg. en que dichos caracteres no están presentes. En sentido contrario opinan autores como Dörfelt (1979), Boekhout \& Bas (1986), Quadraccia \& Lunghini (1990)... etc. que elevan a nivel genérico el rango de los mismos, incluyendo en Oudemansiella s. str. únicamente los táxones no radicantes y con anillo, por lo que todas las especies que aparecen consignadas en este

Comunicación presentada en las XV Jornadas Internacionales de Micología, San Sebastián, 1990. 
artículo habría que incluirlas en Xerula s. str.

Nosotros, con miras a conseguir la mayor simplificación taxonómica posible, utilizaremos el sentido más amplio del género, por lo que incluiremos las cuatro especies tratadas en Oudemansiella Speg.

\section{MATERIAL Y MÉTODOS}

Todo el material estudiado se encuentra depositado en el herbario del Departamento de Biología Vegetal, Facultad de Ciencias (GDAC). Las recolectas analizadas son las siguientes:

Oudemansiella mediterranea (Pacioni \& Lalli) Horak

HUELVA: Coto de Doñana. Arenas litorales. Leg. J.L. Rosúa. 7/XII/1982. GDAC 32621.

Oudemansiella melanotricha (Dörfelt) Moser

CADIZ: Pinar de S. Cristóbal. Tocón de Abies pinsapo Boiss. Leg. G. Moreno et al., 20/XI/1984. GDAC 23690. MALAGA: Sierra de las Nieves, Nava de S. Luis. Raíces de A. pinsapo. Leg. G. Moreno et al., 26/XI/1982. GDAC 16146. Estepona. Los Reales de Genalguacil. Tocón de A. pinsapo. Leg. J. Guerra, 30/XI/1983. GDAC 23682. Yunquera. Mismo hábitat. Leg. G. Moreno et al. 21/XI/1984. GDAC 23791.

Oudemansiella radicata (Relham.: Fr.) Sing.

GRANADA: Alfaguara, Arroyo de Fardes. Tocón de Quercus ilex ssp. ballota. Leg. G. Malençon et al. 17/XI/1977. GDAC 11080. JAÉN: Barranco de Valdeazores. Mismo hábitat. 25/XI/1983. GDAC 23720.

Oudemansiella xeruloides Bon

JAÉN: Mata Bejid. Encinar. 28/XI/1989. GDAC 32622.

La metodología es la clásica en este tipo de estudios, habiendo empleado para el análisis microscópico preferentemente hidróxido potásico al 5\% con miras a la recuperación de material de herbario, así como rojo congo amoniacal para facilitar la observación de estructuras hialinas. Sólo en el caso de O. mediterranea (Pacioni \& Lalli) Horak y O.xeruloides Bon y dado su interés taxonómico y corológico indicaremos el protólogo completo de las mismas.

\section{DISCUSIÓN TAXONÓMICA}

Oudemansiella mediterranea (Pacioni \& Lalli) Horak, Boll. Gruppo Micol. Bresadola 31 (1-2): 34, (1988).

= Hydropus mediterraneus Pacioni \& Lalli

= Xerula mediterranea (Pacioni \& Lalli) Quadraccia

= Flammulina mediterranea (Pacioni \& Lalli) Bas \& Robich.

Este taxon recientemente descubierto y al parecer ampliamente distribuido en 


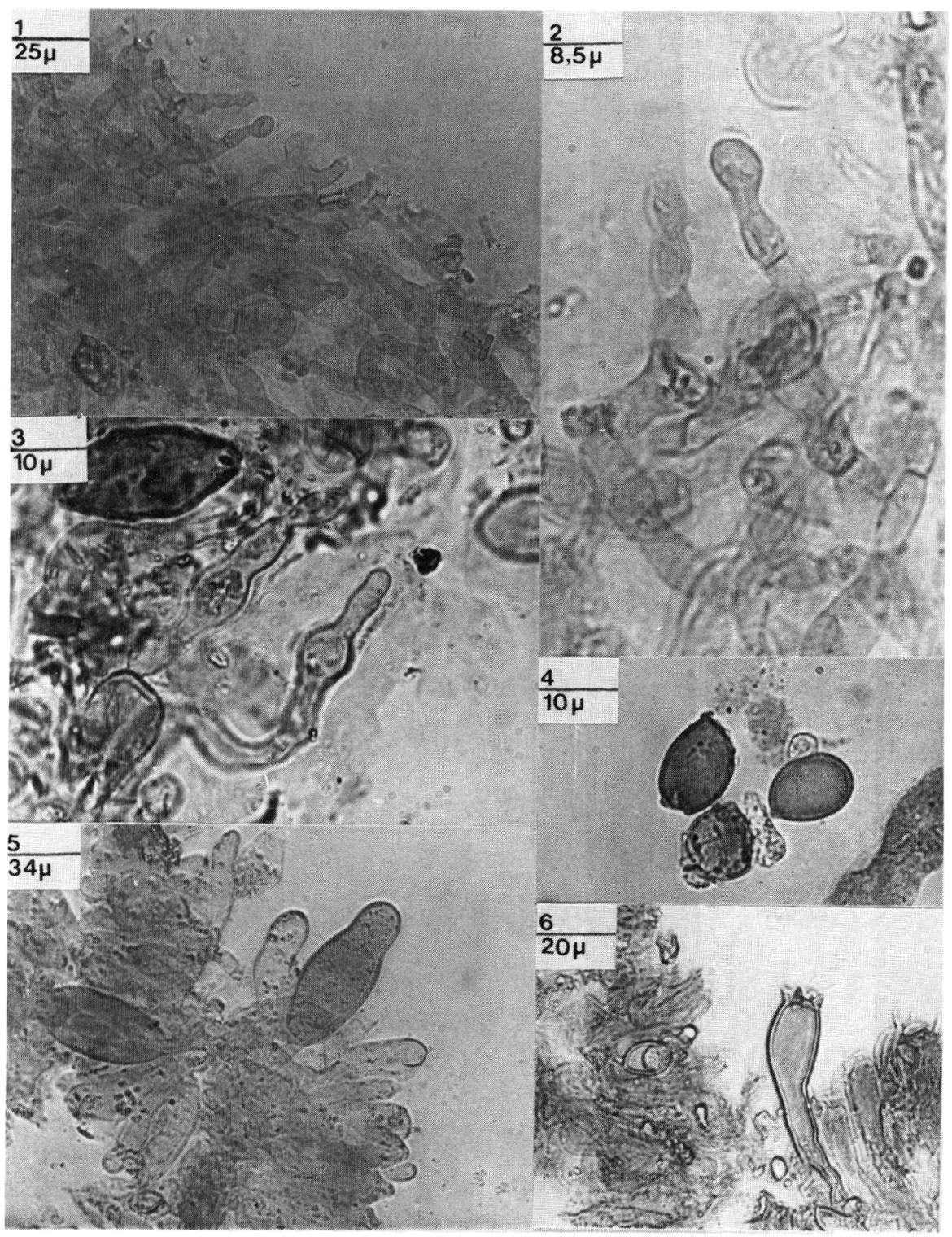

Figs. 1 a 6. Oudemansiella mediterranea: Cutícula: células (1-2); Pelos cuticulares (3); Esporas (4); Pleurocistidios (5); Basidios (6). 
las zonas costeras arenosas de la región mediterránea, se caracteriza fundamentalmente por su cutícula himeniforme constituida por células más o menos claviformes (Fig. 1, 2), entre las que se diferencian largos pelos cilindráceos (Fig. 3), sus basidios tetraspóricos de paredes gruesas (Fig. 6), los cistidios de clavados a fusiformes con el ápice agudo a subcapitado (Fig. 5), así como por sus esporas elipsoides de paredes gruesas midiendo 11-13 x 7-9 $\mu \mathrm{m}$ (Fig. 4).

No obstante, hemos de indicar que el encuadramiento taxonómico de este hongo entraña serias dificultades como ponen de manifiesto las numerosas publicaciones que sobre el mismo han aparecido en los últimos años y en las que se exponen dos hipótesis contrapuestas.

Así, Horak (1988) es partidario de su inclusión en Oudemansiella Speg. (subgénero Xerula (Mre.) Sing.) en base sobre todo a la morfología esporal, basidios metuloides y al aspecto general de los basidiocarpos. Esta misma opinión comparten Quadraccia \& Lunghini (1990), transfiriéndola el primero de ellos al género Xerula Mre.

Contrariamente Bas \& Robich (1988), aunque reconocen las referidas analogías con Xerula, prefieren incluir la especie que nos ocupa en Flammulina Karst. ya que el contex to del estipe no es sarcodimítico (como sucede en la gran mayoría de especies de Xerula, el pigmento de las células del pileipellis no es intracelular y la estructura de la cutícula pileíca no es tan típicamente himeniforme como en las especies del género propuesto por Maire.

Nosotros, analizados con detalle los argumentos aportados por los diferentes autores, y estudiados en profundidad los caracteres de Hydropus mediterraneus Pacioni \& Lalli, pensamos más lógica su inclusión en el género Oudemansiella Speg. en base a los siguientes razonamientos:

a) El hábitat de Oudemansiella mediterranea (Pacioni \& Lalli) Horak al igual que su aspecto general es muy semejante al de algunas otras especies de este género como O. xeruloides Bon (Horak, 1988).

b) Como puede observarse en las figuras correspondientes a los revestimientos pileícos de O. mediterranea (Fig. 1,2,3), O. melanotricha (Fig. 7,8) y $O$. xeruloides (Fig. 12) existe una estrecha relación entre ellos, puesto que en todos los casos son himeniformes, diferenciándose pelos cilindráceos con ápice redondeado o agudo.

c) Los himeniocistidios de las tres especies (Fig. 5, 9, 10, 13) igualmente guardan una estrecha similitud morfológica.

d) Los caulocistidios de O. xeruloides Bon (Fig. 14) son semejantes a los de la especie de Pacioni \& Lalli como se pone de manifiesto si analizamos los esquemas de esta última que aportan Bas \& Robich (1988:492, Fig. 6).

e) Como ya hemos reseñado con anterioridad $O$. mediterranea presenta, al igual que $O$. xeruloides, basidios de paredes gruesas (Fig. 6).

f) Las esporas, aunque de menor talla, son morfológicamente idénticas en $O$. mediterranea (Fig. 4) y O.xeruloides (Fig. 15). 
g) Algunas especies como Xerula kuehneri (Romagn.) Bas \& Boekhout poseen estructura monomítica en la trama del estipe (Boekhout \& Bas, 1986).

h) Como indican Quadraccia \& Lunghini (1990) referenciando a Bas \& Robich (1988), las características de este taxon son aberrantes con respecto a las del resto de especies europeas de Flammulina.

\section{Oudemansiella melanotricha (Dörfelt) Moser}

Desde el punto de vista taxonómico se reconoce con facilidad en base a su cutícula himeniforme constituida por células claviformes a fusiformes, entre las que se diferencian largos pelos de color pardo, de ápice agudo y base ensanchada con paredes engrosadas (Fig. 7, 8). Himenocistidios (Fig. 9, 10) con un ligero engrosamiento parietal, presentando algunos de ellos cristales en su ápice. Esporas (Fig. 11) lisas, subglobosas y midiendo de $8-12 \mu \mathrm{m}$.

Observaciones: Para una mayor información sobre la ecología, corología o taxonomía de este hongo remitimos al lector interesado al trabajo de Moreno et al. (1986).

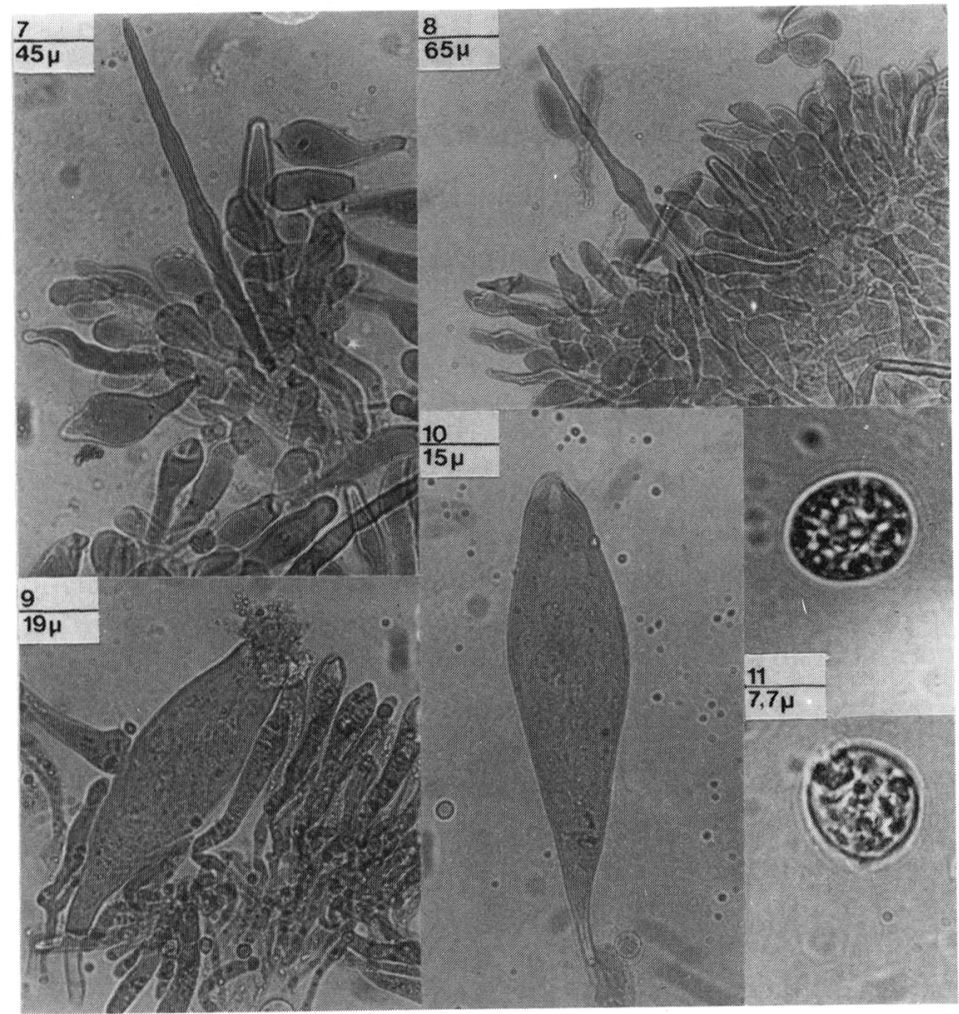

Figs. 7 a 11. Oudemansiella melanotricha: Cutícula (7-8); Cistidios (9-10); Esporas (11). 


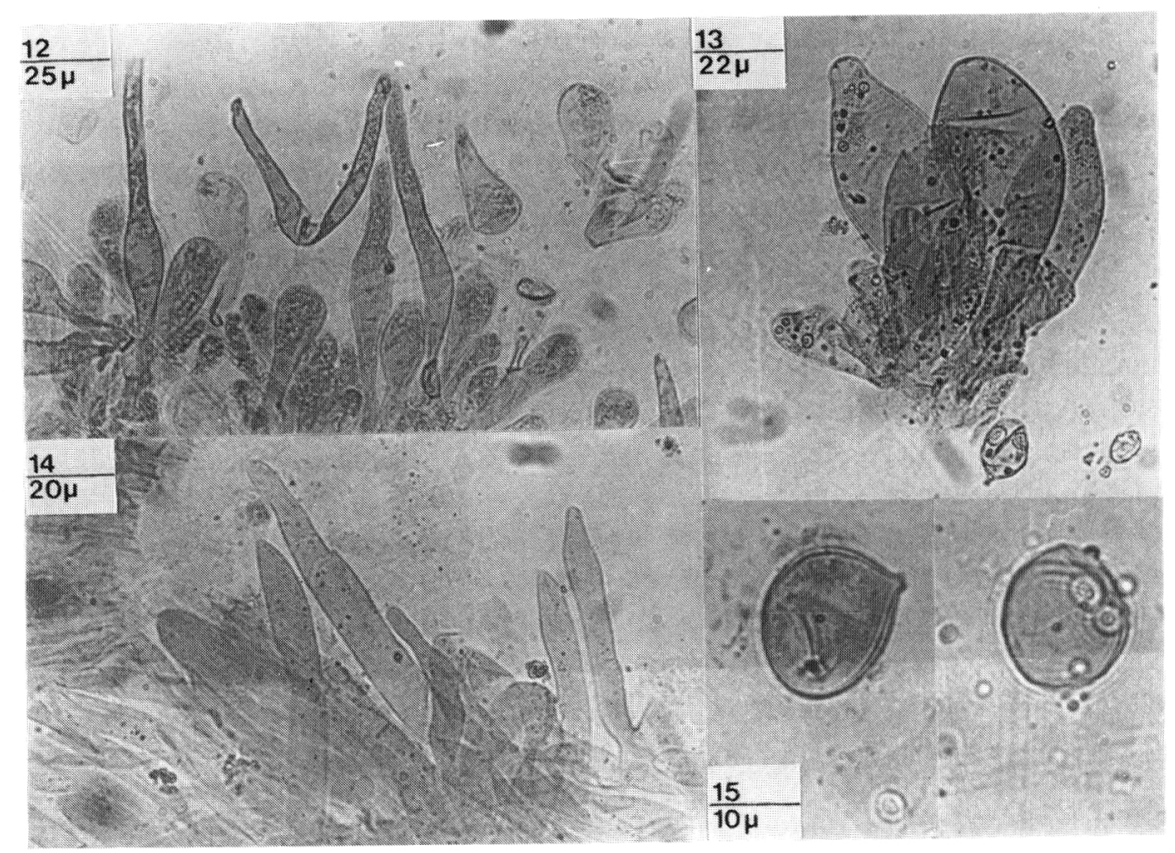

Figs. 12 a 15. Oudemansiella xeruloides: Cutícula (12); Pleurocistidios (13); Caulocistidios (14); Esporas (15).

Oudemansiella radicata (Rehlan.: Fr.) Sing.

Se trata de la especie más frecuente de las cuatro por lo que para un mayor conocimiento de la misma recomendamos consultar (Moreno et al., 1986).

\section{Oudemansiella xeruloides Bon, Doc. Mycol. 4:31 (1975)}

Esta especie fue descrita por Bon en 1975 a partir de material recolectado en la costa francesa, desarrollándose en suelos arenosos en relación con plantas típicamente sabulícolas (Roseto-Ephedretum, Helichrysetum o con Ammophila arenaria). Algunos años después Moreno \& Bon (1985) dan cuenta de la aparición en España de un taxon que asimilan a $O$. xeruloides Bon pero con la que presentan, según los referidos autores, unas claras y constantes diferencias. Este hecho condujo a Moreno et al. (1991) a separar una variedad silvícola cuyas características diferenciales con respecto a la variedad tipo expondremos más adelante.

Pues bien, para complicar aún más las cosas, queremos dar cuenta de la recolección en la provincia de Jaén, en suelo de encinar, de un material que se muestra, en base a sus características macro y microscópicas más próxima a la especie tipo, pero que se desarrolla en ambientes silvícolas.

Veamos ahora algunos de los caracteres más destacables de las diversas recolectas. 
Oudemansiella xeruloides Bon (material francés y británico).

- Sombrerillo plano-convexo de 20-30 mm.

- Pie radicante de 35-50 x 3-5 mm.

- Esporas elipsoidales a subamigdaliformes: 14-16(17) x 10-12(13) $\mu \mathrm{m}$ ó 14$19(21) \times 10-13,5 \mu \mathrm{m}$.

- Hábitat sabulícola.

Oudemansiella xeruloides Bon (material de Jaén).

- Píleo plano convexo de $25 \mathrm{~mm}$.

- Estipe radicante de $35 \times 3 \mathrm{~mm}$.

- Cutícula (Fig. 12) con células piriformes a ovales, entre las que pueden diferenciarse pelos cilindráceos a más o menos sublageniformes.

- Pleurocistidios (Fig. 13) de 65-85 x 20-32 $\mu \mathrm{m}$. Caulocistidios (Fig. 14) de morfología variable.

- Esporas (Fig. 15) elipsoidales a subamigdaliformes de 15-18 x 11-13(-14) $\mu \mathrm{m}$. (Q:L/1:1,23-1,4).

- Hábitat silvícola: Paeonio-Quercetum rotundifoliae.

Oudemansiella xeruloides var. hispanica Moreno, Esteve-Raventós \& Illana.

- Sombrerillo convexo con mamelón marcado, de 30-60 mm.

- Pie radicante de $80-140 \times 3-8 \mathrm{~mm}$.

- Pleurocistidios de 100-180 x 12-30 $\mu \mathrm{m}$.

- Esporas mayormente (sub) amigdaliformes de 16-20 x 11-12 $\mu \mathrm{m}$.

Observaciones: Del análisis de los párrafos precedentes podemos concluir que, si exceptuamos el hábitat, no existen claras diferencias entre las recolectas llevadas a cabo por Bon (1975) y Reid (1985) en el norte de Francia e Inglaterra respectivamente y la obtenida por nosotros mismos en la provincia de Jaén. El problema se plantea al tratar de compararlas con $O$. xeruloides var. hispanica. Sin embargo, si tenemos en cuenta los datos aportados por otros autores (Horak, 1988,...) sobre talla esporal, de los pleurocistidios... etc., podemos concluir que solamente el tamaño de los basidiocarpos separa la variedad propuesta por los micólogos españoles y nuestro material, diferencia de tamaño que puede achacarse simplemente a un diferente grado de desarrollo de los carpóforos o a la influencia de las condiciones ambientales en las que fructifican las distintas poblaciones (Paeonio-Quercetum rotundifolia y Populion albae).

Es por todo lo expuesto por lo que pensamos que efectivamente en comunidades silvícolas se desarrollan poblaciones que taxonómicamente están relacionadas con Oudemansiella xeruloides Bon y de la que son difícilmente separable, a no ser por el ecosistema tan diferente en el que viven. Por tanto, a nuestro modo de ver la separación de ambas sería más lógica a nivel de forma ecológica que de variedad. Sin embargo, desgraciadamente, hasta la fecha no hemos podido realizar más que una sola 
recolección, lo que no hace aconsejable, por el momento, llevar a cabo el cambio de status reseñado mediante el cual el taxon español quedaría definido como O.xeruloides f. hispanica.

AGRADECIMIENTOS. Queremos expresar nuestra gratitud a los Doctores M. Bon (St. Valery sur Sommes, Francia) y G. Moreno (Univ. Alcalá de Henares) la revisión del material de Oudemansiella xeruloides Bon. Así mismo, queremos agradecer a este último la cesión del manuscrito del trabajo de Moreno et al. (1991) referente a O. xeruloides var. hispanica.

\section{BIBLIOGRAFÍA}

BAS, C. \& G. ROBICH -1988- On a false Hydropus, Flammulina mediterranea, comb. nov. Persoonia, 13(4):489-494.

BOEKHOUT, T. \& C. BAS -1986- Notulae and floram Agaricinam Neerlandicam XII. Some notes on the genera Oudemansiella and Xerula. Persoonia, 13(1):45-56.

BON, M. -1975- Agaricales de la côte atlantique française. Doc. Mycol., 4:1-40.

DÖRFELT, H. -1979- Taxonomishe Studien in der Gattung Xerula R. Mre. Reprium nov. Spec. Regni Veg. 90:363-388.

HORAK, E. -1988- Notizie integrative tassonomico-sistematiche su Oudemansiella mediterranea (Pacioni \& Lalli, 1985) comb. nov. Boll. Gruppo Micol. Bresadola XXXI (12):31-37.

MORENO, G. \& M. BON -1985- Nouvelles donnes sur Oudemansiella xeruloides Bon, nouvelle espèce pour l'Espagne. Doc. Mycol., 15(60):39-41.

MORENO, G., J.L.G. MANJÓN y A. ZUGAZA -1986- La guía de Incafo de los hongos de la Península Ibérica. Vol. I y II. Incafo. Madrid.

MORENO, G., F. ESTEVE-RAVENTÓS y C. ILLANA -1991- Some interesting Agarics presented in the Mycological exhibitions of Madrid and Salamanca (Spain) in 1989. Mycologia Helvetica (en prensa).

MOSER, M. -1983- Keys to Agarics and Boleti (Polyporales, Boletales, Agaricales, Russulales) Phillips. London.

PEGLER, D.N.\& T.W.K. YOUNG-1986-Classifications of Oudemansiella (Basidiomycotina, Tricholomataceae) with special reference to spore structure. Trans. Br.Mycol.Soc., 87:583602.

QUADRACCIA, L. \& D. LUNGHINI -1990- Contributo alla conoscenza dei Macromiceti della tenuta presidenziale di Caselporiziano (Micoflora del Lazio II). Accad. Nazionale de Lincei Anno 388, n 264:49-120.

REID, D.A. - 1985- Further records of Jersey fungi. Trans Br. Mycol. Soc., 8:715-722.

(Aceptado para su publicación en abril de 1991)

Dirección de los autores: Departamento de Biología Vegetal. Facultad de Ciencias. Universidad de Granada. 